\title{
Statistical Portfolio Estimation under the Utility Function Depending on Exogenous Variables
}

\author{
Kenta Hamada, Dong Wei Ye, and Masanobu Taniguchi \\ Department of Applied Mathematics, School of Fundamental Science and Engineering, Waseda University, \\ Tokyo 169-8050, Japan \\ Correspondence should be addressed to Masanobu Taniguchi, taniguchi@waseda.jp
}

Received 8 September 2011; Accepted 15 November 2011

Academic Editor: Cathy W. S. Chen

Copyright (C) 2012 Kenta Hamada et al. This is an open access article distributed under the Creative Commons Attribution License, which permits unrestricted use, distribution, and reproduction in any medium, provided the original work is properly cited.

In the estimation of portfolios, it is natural to assume that the utility function depends on exogenous variable. From this point of view, in this paper, we develop the estimation under the utility function depending on exogenous variable. To estimate the optimal portfolio, we introduce a function of moments of the return process and cumulant between the return processes and exogenous variable, where the function means a generalized version of portfolio weight function. First, assuming that exogenous variable is a random process, we derive the asymptotic distribution of the sample version of portfolio weight function. Then, an influence of exogenous variable on the return process is illuminated when exogenous variable has a shot noise in the frequency domain. Second, assuming that exogenous variable is nonstochastic, we derive the asymptotic distribution of the sample version of portfolio weight function. Then, an influence of exogenous variable on the return process is illuminated when exogenous variable has a harmonic trend. We also evaluate the influence of exogenous variable on the return process numerically.

\section{Introduction}

In the usual theory of portfolio analysis, optimal portfolios are determined by the mean $\mu$ and the variance $\Sigma$ of the portfolio return $X=\{X(t)\}$. Several authors proposed estimators of optimal portfolios as functions of the sample mean $\widehat{\mu}$ and the sample variance $\widehat{\Sigma}$ for independent returns of assets. However, empirical studies show that financial return processes are often dependent and non-Gaussian. Shiraishi and Taniguchi [1] showed that the above estimators are not asymptotically efficient generally if the returns are dependent. Under the nonGaussianity, if we consider a general utility function $U(\cdot)$, the expected utility should depend on higher-order moments of the return. From this point of view, Shiraishi and Taniguchi [1] proposed the portfolios including higher-order moments of the return.

However, empirical studies show that the utility function often depends on exogenous variable $Z=\{Z(t)\}$. From this point of view, in this paper, we develop the estimation under 
the utility function depending on exogenous variable. Denote the optimal portfolio estimator by a function $\widehat{g}=g(\widehat{\theta})=g(\widehat{\mathbb{E}}(X), \widehat{\operatorname{cov}}(X, X), \widehat{\operatorname{cov}}(X, Z), \widehat{\operatorname{cum}}(X, X, Z))$ where hat $(\widehat{\bullet})$ means the sample version of $(\cdot)$. Although Shiraishi and Taniguchi's [1] setting does not include the exogenous variable $Z(t)$ in $\hat{g}$, we can develop the asymptotic theory in the light of their work.

First, assuming that $Z=\{Z(t)\}$ is a random process, we derive the asymptotic distribution of $\hat{g}$. Then, an influence of $Z$ on the return process is illuminated when $Z$ has a shot noise in the frequency domain. Second, assuming that $Z$ is a nonrandom sequence of variables which satisfy Grenander's conditions, we also derive the asymptotic distribution of $\hat{g}$. Then an influence of $Z$ on $X$ is evaluated when $Z$ is a sequence of harmonic functions. Numerical studies will be given, and they show some interesting features.

The paper is organized as follows. Section 2 introduces the optimal portfolio of the form $\hat{g}$ and provides the asymptotic distribution of $\hat{g}$. Assuming that $Z$ is a stochastic process, we derive the asymptotics of $\widehat{g}$ when $Z$ has a shot noise in the frequency domain. The influence of $Z$ on $X$ is numerically evaluated in Section 2.2. Assuming that $Z$ is a nonrandom sequence satisfying Grenander's conditions, we derive the asymptotic distribution of $\hat{g}$. Section 3 provides numerical studies for the influence of $Z$ on $X$ when $Z$ is a sequence of harmonic functions. The appendix gives the proofs of all the theorems.

\section{Optimal Portfolio with the Exogenous Variables}

Suppose the existence of a finite number of assets indexed by $i,(i=1, \ldots, p)$. Let $X(t)=\left(X_{1}(t), \ldots, X_{p}(t)\right)^{\prime}$ denote the random returns on $p$ assets at time $t$, and let $Z(t)=$ $\left(Z_{1}(t), \ldots, Z_{q}(t)\right)^{\prime}$ denote the exogenous variables influencing on the utility function at time $t$. We write $Y(t)=\left(X(t)^{\prime}, Z(t)^{\prime}\right)^{\prime}=\left(X_{1}(t), \ldots, X_{p}(t), Z_{1}(t), \ldots, Z_{q}(t)\right)^{\prime}$.

Since it is empirically observed that $\{X(t)\}$ is non-Gaussian and dependent, we will assume that it is a non-Gaussian stationary process with the $3^{\text {rd }}$-order cumulants. Also, suppose that there exists a risk-free asset whose return is denoted by $Y_{0}(t)$. Let $\alpha_{0}$ and $\alpha=(\overbrace{\alpha_{1}, \ldots, \alpha_{p}}^{p}$ $\overbrace{0, \ldots, 0}^{q})^{\prime}$ be the portfolio weights at time $t$, and the portfolio is $M(t)=Y(t)^{\prime} \alpha+Y_{0}(t) \alpha_{0}$ whose higher-order cumulants are written as

$$
\begin{aligned}
& c_{1}^{M}(t)=\operatorname{cum}\{M(t)\}=c^{a_{1}} \alpha_{a_{1}}+Y_{0}(t) \alpha_{0} \\
& c_{2}^{M}(t)=\operatorname{cum}\{M(t), M(t)\}=c^{a_{2} a_{3}} \alpha_{a_{2}} \alpha_{a_{3}} \\
& c_{3}^{M}(t)=\operatorname{cum}\{M(t), M(t), M(t)\}=c^{a_{4} a_{5} a_{6}} \alpha_{a_{4}} \alpha_{a_{5}} \alpha_{a_{6}} .
\end{aligned}
$$

We use Einstein's summation convention here and throughout the paper. For a utility function $U(\cdot)$, the expected utility can be approximated as

$$
\mathbb{E}[U(M(t))] \approx U\left(c_{1}^{M}(t)\right)+\frac{1}{2 !} D^{2} U\left(c_{1}^{M}(t)\right) c_{2}^{M}(t)+\frac{1}{3 !} D^{3} U\left(c_{1}^{M}(t)\right) c_{3}^{M}(t)
$$


by Taylor expansion of order 3. The approximate optimal portfolio may be described as

$$
\begin{aligned}
\max _{\alpha_{0}, \alpha} & \{\text { the right hand side of }(2.2)\}, \\
\text { subject to } & \alpha_{0}+\sum_{i=1}^{p} \alpha_{i}=1 .
\end{aligned}
$$

Solving (2.4), Shiraishi and Taniguchi [1] introduced the optimal portfolio depending on the mean, variance, and the third-order cumulants, and then derived the asymptotic distribution of a sample version estimator. Although our problem is different from that of Shiraishi and Taniguchi [1], we develop the discussion with the methods inspired by them.

Introduce a portfolio estimator function based on observed higher-order cumulants,

$$
\widehat{g} \equiv g(\widehat{\theta})=g(\widehat{\mathbb{E}}(X), \widehat{\operatorname{cov}}(X, X), \widehat{\operatorname{cov}}(X, Z), \widehat{\operatorname{cum}}\{X, X, Z\}),
$$

and assume that the function $g(\cdot)$ is $p$-dimensional and measurable, that is,

$$
g(\theta): g(\mathbb{E}(X), \operatorname{cov}(X, X), \operatorname{cov}(X, Z), \operatorname{cum}\{X, X, Z\}) \longrightarrow \mathbf{R}^{p}
$$

Let the random process $\left\{\mathbf{Y}(t)=\left(Y_{1}(t), \ldots, Y_{p+q}(t)\right)^{\prime}\right\}$ be a $(p+q)$-vector linear process generated by

$$
\mathbf{Y}(t)=\sum_{j=0}^{\infty} G(j) \varepsilon(t-j)+\boldsymbol{\mu}
$$

where $\{\varepsilon(t)\}$ is a $(p+q)$-dimensional stationary process such that $\mathbb{E}\{\varepsilon(t)\}=0$ and $\mathbb{E}\left\{\varepsilon(s) \varepsilon(t)^{\prime}\right\}=\delta(s, t) K$, with $K$ a nonsingular $(p+q) \times(p+q)$-matrix, $G(j)^{\prime}$ s are $(p+q) \times(p+q)$ matrices, and $\mu=\left(\mu_{1}, \ldots, \mu_{p+q}\right)$ is the mean vector of $\{\mathbf{Y}(t)\}$. All the components of $\mathbf{Y}, \varepsilon, G, \boldsymbol{\mu}$ are real. Assuming that $\{\varepsilon(t)\}$ has all order cumulants, let $Q_{a_{1}, \ldots, a_{j}}^{e}\left(t_{1}, \ldots, t_{j-1}\right)$ be the joint $j$ thorder cumulant of $\varepsilon_{a_{1}}(t), \varepsilon_{a_{2}}\left(t+t_{1}\right), \ldots, \varepsilon_{a_{j}}\left(t+t_{j-1}\right)$. In what follows we assume that, for each $j=1,2,3, \ldots$,

$$
\begin{gathered}
\sum_{t_{1}, \ldots, t_{j}=-\infty}^{\infty} \sum_{a_{1}, \ldots, a_{j}=1}^{p+q}\left|Q_{a_{1}, \ldots, a_{j}}^{e}\left(t_{1}, \ldots, t_{j-1}\right)\right|<\infty, \\
\sum_{t=0}^{\infty}\|G(t)\|<\infty .
\end{gathered}
$$

Letting $Q_{a_{1}, \ldots, a_{j}}^{Y}\left(t_{1}, \ldots, t_{j-1}\right)$ be the joint $j$ th-order cumulant of $Y_{a_{1}}(t), Y_{a_{2}}(t+$ $\left.t_{1}\right), \ldots, Y_{a_{j}}\left(t+t_{j-1}\right)$, we define the $j$ th-order cumulant spectral density by

$$
\begin{aligned}
f_{a_{1}, \ldots, a_{j}}\left(\lambda_{1}, \ldots, \lambda_{j-1}\right)=\left(\frac{1}{2 \pi}\right)^{j-1} \sum_{t_{1}, \ldots, t_{j-1}=-\infty}^{\infty} & \exp \left\{-i\left(\lambda_{1} t_{1}+\cdots+\lambda_{j-1} t_{j-1}\right)\right\} \\
\times & Q_{a_{1}, \ldots, a_{j}}^{Y}\left(t_{1}, \ldots, t_{j-1}\right),
\end{aligned}
$$


which is expressed as

$$
\begin{aligned}
& f_{a_{1}, \ldots, a_{j}}\left(\lambda_{1}, \ldots, \lambda_{j-1}\right) \\
& \quad=\sum_{b_{1}, \ldots, b_{j}=1}^{p+q} k_{a_{1} b_{1}}\left(\lambda_{1}+\cdots+\lambda_{j-1}\right) k_{a_{2} b_{2}}\left(-\lambda_{1}\right) \cdots k_{a_{j-1} b_{j-1}}\left(-\lambda_{j-2}\right) k_{a_{j} b_{j}}\left(-\lambda_{j-1}\right) \widetilde{Q}_{a_{1}, \ldots, a_{j}}^{e}\left(\lambda_{1}, \ldots, \lambda_{j-1}\right),
\end{aligned}
$$

where $\tilde{Q}_{a_{1}, \ldots, a_{j}}^{e}$ is the $j$ th-order cumulant spectral density of $\varepsilon_{a_{1}}(t), \ldots, \varepsilon_{a_{j}}(t), k_{a b}(\lambda)=$ $\sum_{l=0}^{\infty} G_{a b}(l) e^{i \lambda l}$ and $G_{a b}(l)$ is the $(a, b)$ th element of $G(l)$. We introduce the following quantities:

$$
\begin{gathered}
\widehat{c}^{a_{1}}=\frac{1}{n} \sum_{s=1}^{n} Y_{a_{1}}(s), \\
\widehat{c}^{a_{2} a_{3}}=\frac{1}{n} \sum_{s=1}^{n}\left(Y_{a_{2}}(s)-\widehat{c}^{a_{2}}\right)\left(Y_{a_{3}}(s)-\widehat{c}^{a_{3}}\right), \\
\widehat{c}^{a_{4} a_{5}}=\frac{1}{n} \sum_{s=1}^{n}\left(Y_{a_{4}}(s)-\widehat{c}^{a_{4}}\right)\left(Y_{a_{5}}(s)-\widehat{c}^{a_{5}}\right), \\
\widehat{c}^{a_{6} a_{7} a_{8}}=\frac{1}{n} \sum_{s=1}^{n}\left(Y_{a_{6}}(s)-\widehat{c}^{a_{6}}\right)\left(Y_{a_{7}}(s)-\widehat{c}^{a_{7}}\right)\left(Y_{a_{8}}(s)-\widehat{c}^{a_{8}}\right),
\end{gathered}
$$

where $1 \leq a_{1}, a_{2}, a_{3}, a_{4}, a_{6}, a_{7} \leq p$ and $p+1 \leq a_{5}, a_{8} \leq p+q$. Write the quantities that appeared in (2.4) by

$$
\begin{aligned}
& \widehat{\theta}=\left(\widehat{c}^{a_{1}}, \widehat{c}^{a_{2} a_{3}}, \widehat{c}^{a_{4} a_{5}}, \widehat{c}^{a_{6} a_{7} a_{8}}\right), \\
& \theta=\left(c^{a_{1}}, c^{a_{2} a_{3}}, c^{a_{4} a_{5}}, c^{a_{6} a_{7} a_{8}}\right),
\end{aligned}
$$

where $c^{a_{1}, \ldots, a_{j}} \equiv Q_{a_{1}, \ldots, a_{j}}^{Y}(0, \ldots, 0)$. Then $\operatorname{dim} \theta=\operatorname{dim} \widehat{\theta}=a+b+c+d$, where $a=p, b=p(p+1) / 2$, $c=p q, d=p(p+1) q / 2$.

First, we derive the asymptotics of the fundamental quantity $\widehat{\theta}$.

Theorem 2.1. Under the assumptions,

$$
\sqrt{n}(\widehat{\theta}-\theta) \stackrel{\oplus}{\longrightarrow} N(0, \Omega), \quad(n \longrightarrow \infty),
$$

where

$$
\Omega=\left(\begin{array}{llll}
\Omega_{11} & \Omega_{12} & \Omega_{13} & \Omega_{14} \\
\Omega_{21} & \Omega_{22} & \Omega_{23} & \Omega_{24} \\
\Omega_{31} & \Omega_{32} & \Omega_{33} & \Omega_{34} \\
\Omega_{41} & \Omega_{42} & \Omega_{43} & \Omega_{44}
\end{array}\right),
$$


and the typical element of $\Omega_{i j}$ corresponding to the covariance between $\widehat{c}^{\Delta}$ and $\widehat{c}^{\nabla}$ is denoted by $V\{(\Delta)(\nabla)\}$, and

$$
\begin{aligned}
& V\left\{\left(a_{1}\right)\left(a_{1}^{\prime}\right)\right\}=\Omega_{11}=2 \pi f_{a_{1} a_{1}^{\prime}}(0), \\
& V\left\{\left(a_{2} a_{3}\right)\left(a_{1}^{\prime}\right)\right\}=\Omega_{12}=2 \pi \int_{-\pi}^{\pi} f_{a_{2} a_{3} a_{1}^{\prime}}(\lambda, 0) d \lambda\left(=\Omega_{21}\right), \\
& V\left\{\left(a_{4} a_{5}\right)\left(a_{1}^{\prime}\right)\right\}=\Omega_{13}=2 \pi \int_{-\pi}^{\pi} f_{a_{4} a_{5} a_{1}^{\prime}}(\lambda, 0) d \lambda\left(=\Omega_{31}\right), \\
& V\left\{\left(a_{6} a_{7} a_{8}\right)\left(a_{1}^{\prime}\right)\right\}=\Omega_{14}=2 \pi \iint_{-\pi}^{\pi} f_{a_{6} a_{7} a_{8} a_{1}^{\prime}}\left(\lambda_{1}, \lambda_{2}, 0\right) d \lambda_{1} d \lambda_{2}\left(=\Omega_{41}\right), \\
& V\left\{\left(a_{2} a_{3}\right)\left(a_{2}^{\prime} a_{3}^{\prime}\right)\right\}=\Omega_{22}=2 \pi \iint_{-\pi}^{\pi} f_{a_{2} a_{3} a_{2}^{\prime} a_{3}^{\prime}}\left(\lambda_{1}, \lambda_{2},-\lambda_{2}\right) d \lambda_{1} d \lambda_{2} \\
& +2 \pi \int_{-\pi}^{\pi}\left\{f_{a_{2} a_{2}^{\prime}}(\lambda) f_{a_{3} a_{3}^{\prime}}(-\lambda)+f_{a_{2} a_{3}^{\prime}}(\lambda) f_{a_{3} a_{2}^{\prime}}(-\lambda)\right\} d \lambda, \\
& V\left\{\left(a_{2} a_{3}\right)\left(a_{4}^{\prime} a_{5}^{\prime}\right)\right\}=\Omega_{23}=2 \pi \iint_{-\pi}^{\pi} f_{a_{2} a_{3} a_{4}^{\prime} a_{5}^{\prime}}\left(\lambda_{1}, \lambda_{2},-\lambda_{2}\right) d \lambda_{1} d \lambda_{2} \\
& +2 \pi \int_{-\pi}^{\pi}\left\{f_{a_{2} a_{4}^{\prime}}(\lambda) f_{a_{3} a_{5}^{\prime}}(-\lambda)+f_{a_{2} a_{5}^{\prime}}(\lambda) f_{a_{3} a_{4}^{\prime}}(-\lambda)\right\} d \lambda\left(=\Omega_{32}\right), \\
& V\left\{\left(a_{2} a_{3}\right)\left(a_{6}^{\prime} a_{7}^{\prime} a_{8}^{\prime}\right)\right\}=\Omega_{24}=2 \pi \iiint_{-\pi}^{\pi} f_{a_{2} a_{3} a_{6}^{\prime} a_{7}^{\prime} a_{8}^{\prime}}\left(\lambda_{1}, \lambda_{2}, \lambda_{3},-\lambda_{3}\right) d \lambda_{1} d \lambda_{2} d \lambda_{3}\left(=\Omega_{42}\right), \\
& V\left\{\left(a_{4} a_{5}\right)\left(a_{4}^{\prime} a_{5}^{\prime}\right)\right\}=\Omega_{33}=2 \pi \iint_{-\pi}^{\pi} f_{a_{4} a_{5} a_{4}^{\prime} a_{5}^{\prime}}\left(\lambda_{1}, \lambda_{2},-\lambda_{2}\right) d \lambda_{1} d \lambda_{2} \\
& +2 \pi \int_{-\pi}^{\pi}\left\{f_{a_{4} a_{4}^{\prime}}(\lambda) f_{a_{5} a_{5}^{\prime}}(-\lambda)+f_{a_{4} a_{5}^{\prime}}(\lambda) f_{a_{5} a_{4}^{\prime}}(-\lambda)\right\} d \lambda, \\
& V\left\{\left(a_{2} a_{3}\right)\left(a_{6}^{\prime} a_{7}^{\prime} a_{8}^{\prime}\right)\right\}=\Omega_{34}=2 \pi \iiint_{-\pi}^{\pi} f_{a_{4} a_{5} a_{6}^{\prime} a_{7}^{\prime} a_{8}^{\prime}}\left(\lambda_{1}, \lambda_{2}, \lambda_{3},-\lambda_{3}\right) d \lambda_{1} d \lambda_{2} d \lambda_{3}\left(=\Omega_{43}\right), \\
& V\left\{\left(a_{6} a_{7} a_{8}\right)\left(a_{6}^{\prime} a_{7}^{\prime} a_{8}^{\prime}\right)\right\}=\Omega_{44}=2 \pi \iiint \int_{-\pi}^{\pi} f_{a_{6} a_{7} a_{8} a_{6}^{\prime} a_{7}^{\prime} a_{8}^{\prime}}\left(\lambda_{1}, \lambda_{2}, \lambda_{3},-\lambda_{3}-\lambda_{4}\right) d \lambda_{1} \cdots d \lambda_{4} \\
& +2 \pi \iiint_{-\pi}^{\pi} \sum_{v_{1}} f_{a_{i_{1}} a_{i_{2}} a_{i_{3}} a_{i_{4}}}\left(\lambda_{1}, \lambda_{2}, \lambda_{3}\right) f_{a_{i_{5}} a_{i_{6}}} \\
& \times\left(-\lambda_{2}-\lambda_{3}\right) d \lambda_{1} d \lambda_{2} d \lambda_{3} \\
& +2 \pi \iiint_{-\pi}^{\pi} \sum_{v_{2}} f_{a_{i_{1}} a_{i_{2}} a_{i_{3}}}\left(\lambda_{1}, \lambda_{2}\right) f_{a_{i_{4}} a_{i_{5}} a_{i_{6}}} \\
& \times\left(\lambda_{3},-\lambda_{2}-\lambda_{3}\right) d \lambda_{1} d \lambda_{2} d \lambda_{3} \\
& +2 \pi \iiint_{-\pi}^{\pi} \sum_{\nu_{3}} f_{a_{i_{1}} a_{i_{2}}}\left(\lambda_{1}\right) f_{a_{i_{3}} a_{i_{4}}}\left(\lambda_{2}\right) f_{a_{i_{5}} a_{i_{6}}}\left(-\lambda_{1}-\lambda_{2}\right) d \lambda_{1} d \lambda_{2} \text {. }
\end{aligned}
$$


Table 1: Standardized 3rd-order cumulants of the returns of five stocks from 2005/11/08 to 2011/11/08.

\begin{tabular}{lccccc}
\hline & IBM & Ford & Merck & HP & EXXON \\
\hline$\widehat{s}$ & 0.05608 & 2.50879 & 0.79521 & 9.84777 & 0.34485 \\
\hline
\end{tabular}

In what follows we place all the proofs of theorems in the appendix.

Next we discuss the estimation of portfolio $g(\theta)$. For this we assume that the portfolio function $g(\theta)$ is continuously differentiable. Henceforth, we use a unified estimator $g(\widehat{\theta})$ for $g(\theta)$. The $\delta$-method and Slutsky's lemma imply the following.

Theorem 2.2. Under the assumptions

$$
\sqrt{n}(g(\widehat{\theta})-g(\theta)) \stackrel{\oplus}{\longrightarrow} N\left(\mathbf{0},(D g) \Omega(D g)^{\prime}\right), \quad(n \longrightarrow \infty)
$$

where, $D g=\left\{\partial_{i} g^{j} ; i=1, \ldots, \operatorname{dim} \theta, j=1, \ldots, p+q\right\}$.

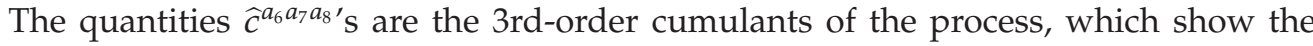
non-Gaussianity. For the returns of five financial stocks IBM, Ford, Merck, HP, and EXXON, we calculated the standardized 3rd-order cumulants $\widehat{s}=\widehat{c}^{a_{6} a_{7} a_{8}} /\left(\widehat{v}^{2}\right)^{3 / 2}$ where $\widehat{v}^{2}$ is the sample variance of the stock. Table 1 below shows their values.

From Table 1 we observe that the five returns are non-Gaussian. In view of Theorem 2.1, it is possible to construct the $(1-\alpha)$ confidence interval for $c=c^{a_{6} a_{7} a_{8}}$ in the following form:

$$
\left[\widehat{c}-\frac{z_{\alpha}}{\sqrt{n}} \widehat{\Omega}_{44}^{1 / 2}, \widehat{c}+\frac{z_{\alpha}}{\sqrt{n}} \widehat{\Omega}_{44}^{1 / 2}\right]
$$

where $z_{\alpha}$ is the upper level- $\alpha$ point of $N(0,1)$ and $\widehat{\Omega}_{44}$ is a consistent estimator of $\Omega_{44}$ calculated by the method of Keenan [2] and Taniguchi [3].

\subsection{Influence of Exogenous Variable}

In this subsection we investigate an influence of the exogenous variables $Z(t)$ on the asymptotics of the portfolio estimator $g(\widehat{\theta})$.

Assume that the exogenous variables have "shot noise" in the frequency domain, that is,

$$
Z_{a_{j}}(\lambda)=\delta\left(\lambda_{a_{j}}-\lambda\right)
$$

where $\delta(\cdot)$ is the Dirac delta function with period $2 \pi$, and $\lambda_{a_{j}} \neq 0$, hence $Z_{a_{j}}(\lambda)$ has one peak at $\lambda+\lambda_{a_{j}} \equiv 0(\bmod 2 \pi)$. 
Theorem 2.3. For (2.17), denote $\Omega_{i j}$ and $V\{(\Delta)(\nabla)\}$ in Theorem 2.1 by $\Omega_{i j}^{\prime}$ and $V^{\prime}\{(\Delta)(\nabla)\}$, respectively. That is, $\Omega_{i j}^{\prime}$ and $V^{\prime}\{(\Delta)(\nabla)\}$ represent the asymptotic variance when the exogenous variables are shot noise. Then,

$$
\begin{aligned}
& V^{\prime}\left\{\left(a_{4} a_{5}\right)\left(a_{1}^{\prime}\right)\right\}=\Omega_{13}^{\prime}= 0\left(=\Omega_{31}^{\prime}\right), \\
& V^{\prime}\left\{\left(a_{6} a_{7} a_{8}\right)\left(a_{1}^{\prime}\right)\right\}=\Omega_{14}^{\prime}= 2 \pi f_{a_{6} a_{7} a_{1}^{\prime}}\left(\lambda_{a_{8}}, 0\right)\left(=\Omega_{41}^{\prime}\right), \\
& V^{\prime}\left\{\left(a_{2} a_{3}\right)\left(a_{4}^{\prime} a_{5}^{\prime}\right)\right\}=\Omega_{23}^{\prime}=2 \pi f_{a_{2} a_{3} a_{4}^{\prime}}\left(\lambda_{a_{5}^{\prime}}, 0\right)+2 \pi f_{a_{2} a_{4}^{\prime}}\left(\lambda_{a_{5}}\right) f_{a_{3} a_{5}^{\prime}}\left(-\lambda_{a_{5}^{\prime}}\right) \\
&+ 2 \pi f_{a_{2} a_{5}^{\prime}}\left(-\lambda_{a_{5}^{\prime}}\right) f_{a_{3} a_{4}^{\prime}}\left(\lambda_{a_{5}}\right)\left(=\Omega_{32}^{\prime}\right), \\
& V^{\prime}\left\{\left(a_{2} a_{3}\right)\left(a_{6}^{\prime} a_{7}^{\prime} a_{8}^{\prime}\right)\right\}=\Omega_{24}^{\prime}=2 \pi \iint_{-\pi}^{\pi} f_{a_{2} a_{3} a_{6}^{\prime} a_{7}^{\prime} a_{8}^{\prime}}\left(-\lambda_{a_{8}^{\prime}}, \lambda_{1}, \lambda_{2},-\lambda_{2}\right) d \lambda_{1} d \lambda_{2}\left(=\Omega_{42}^{\prime}\right), \\
& V^{\prime}\left\{\left(a_{4} a_{5}\right)\left(a_{4}^{\prime} a_{5}^{\prime}\right)\right\}=\Omega_{33}^{\prime}=2 \pi f_{a_{4} a_{5} a_{4}^{\prime} a_{5}^{\prime}}\left(\lambda_{a_{5}^{\prime}}, \lambda_{a_{5}},-\lambda_{a_{5}}\right)+2 \pi f_{a_{4} a_{4}^{\prime}}\left(\lambda_{a_{5}}\right) f_{a_{5} a_{5}^{\prime}}\left(-\lambda_{a_{5}}\right) \\
&+2 \pi f_{a_{4} a_{5}^{\prime}}\left(\lambda_{a_{5}}\right) f_{a_{5} a_{4}^{\prime}}\left(-\lambda_{a_{5}}\right), \\
& V^{\prime}\left\{\left(a_{4} a_{5}\right)\left(a_{1}{ }^{\prime} a_{1}^{\prime}\right)\right\}=\Omega_{34}^{\prime}= 2 \pi \int_{-\pi}^{\pi} f_{a_{4} a_{5} a_{6}^{\prime} a_{7}^{\prime} a_{8}^{\prime}}\left(-\lambda_{a_{8}^{\prime}}, \lambda_{a_{5}}, \lambda,-\lambda\right) d \lambda\left(=\Omega_{43}^{\prime}\right) .
\end{aligned}
$$

\subsection{Numerical Studies for Stochastic Exogenous Variables}

This subsection provides some numerical examples which show the influence of $Z(t)$ on $\Omega_{i j}$.

Example 2.4. For a risk-free asset $X_{0}(t)$ and risky asset $X(t)$, we consider construction of optimal portfolios $\alpha X(t)+\alpha_{0} X_{0}(t)$. Here $\{X(t)\}$ is the return process of the risky asset, which is generated by

$$
X(t)=\theta X(t-1)+\varepsilon(t)+\mu_{1}
$$

where $\mathbb{E}\{\varepsilon(t)\}=0, \operatorname{Var}\{\varepsilon(t)\}=\sigma^{2}$. We assume that $X_{0}(t)=\mu$, and that the exogenous variable in the frequency domain is given by $Z(\lambda)=\delta(\lambda)$. Write,

$$
Y(t)=\left(X(t) X_{0}(t) Z(t)^{\prime}\right)
$$

then

$$
\begin{aligned}
\Omega_{13}^{\prime}=\Omega_{31}^{\prime} & =V^{\prime}\left\{\left(a_{4} a_{5}\right)\left(a_{1}^{\prime}\right)\right\}=0, \\
\Omega_{23}^{\prime}=\Omega_{32}^{\prime} & =V^{\prime}\left\{\left(a_{2} a_{3}\right)\left(a_{4}^{\prime} a_{5}^{\prime}\right)\right\}=0, \\
\Omega_{33}^{\prime} & =V\left\{\left(a_{4} a_{5}\right)\left(a_{4}^{\prime} a_{5}^{\prime}\right)\right\} \\
& =\operatorname{cum}\left(a_{1}, a_{1}^{\prime}\right) \operatorname{cum}\left(a_{3}, a_{3}^{\prime}\right) \\
& =\sigma^{2} \frac{1}{\left(1-\theta e^{i \lambda_{a_{3}}}\right)}
\end{aligned}
$$




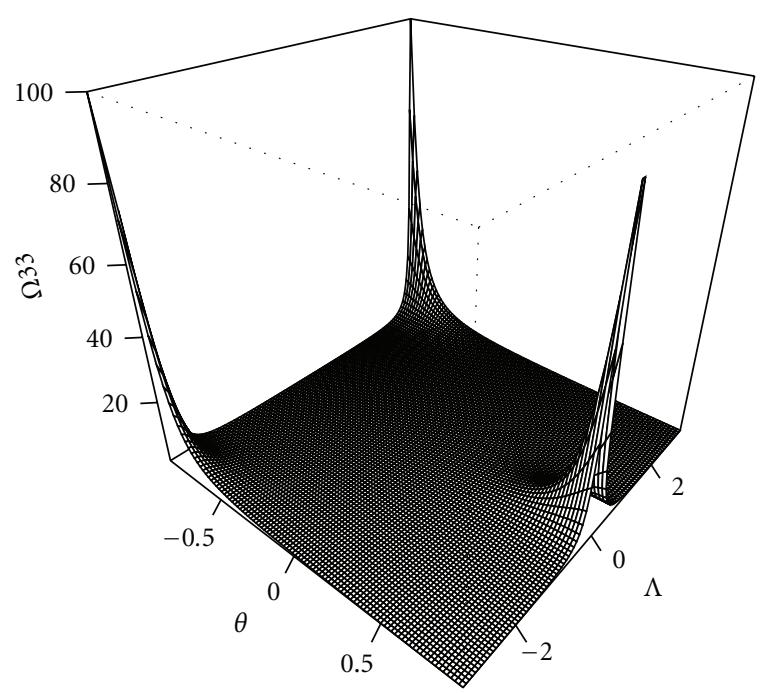

Figure 1: Values of $\Omega_{33}^{\prime}$ for $\theta=-0.9(0.018) 0.9, \lambda_{a_{3}}=-\pi(0.06) \pi$.

which are covarances between $Z(t)$ and $X(t)$, and show an influence $Z(t)$ on $X(t)$. From Figure 1 , it is seen that as $\theta$ tends to 1 , and $\lambda_{a_{3}}$ tends to 0 , then $\Omega_{33}^{\prime}$ increases. If $\theta$ tends to -1 and $\lambda_{a_{3}}$ tends to $-\pi, \pi$, then $\Omega_{33}^{\prime}$ also increases, which entails that the exogenous variables have big influence on the asymptotics of estimators when $\theta$ is close to the unit root of $\operatorname{AR}(2.2)$.

Remark 2.5. $\Omega_{13}^{\prime}$ is robust for the shot noise in $Z(t)$ at $\lambda=\lambda_{a_{3}}$.

\section{Portfolio Estimation for Nonstochastic Exogenous Variables}

So far we assumed that the sequence of exogenous variables $\{Z(t)\}$ is a random stochastic process. In this section, assuming that $\{Z(t)\}$ is a nonrandom sequence, we will propose a portfolio estimator, and elucidate the asymptotics. We introduce the following quantities,

$$
\begin{aligned}
\widehat{A}_{j, k} & =\frac{\sum_{t=1}^{n} X_{j}(t) Z_{k}(t)}{\sqrt{n \sum_{t=1}^{n} Z_{k}^{2}(t)}}, \\
\widehat{B}_{j, m, k} & =\frac{\sum_{t=1}^{n} X_{j}(t) X_{m}(t) Z_{k}(t)}{\sqrt{n \sum_{t=1}^{n} Z_{k}^{2}(t)}} .
\end{aligned}
$$

We assume that $Z(t)$ 's satisfy Grenander's conditions (G1)-(G4) with

$$
a_{j, k}^{(n)}(h)=\sum_{t=1}^{n-h} Z_{j}(t+h) Z_{k}(t) .
$$

(G1) $\lim _{n \rightarrow \infty} a_{j, j}^{(n)}(0)=\infty,(j=1, \ldots, q)$.

(G2) $\lim _{n \rightarrow \infty} Z_{j}(n+1)^{2} / a_{j, j}^{(n)}(0)=0,(j=1, \ldots, q)$. 
(G3) $a_{j, k}^{(n)}(h) / \sqrt{a_{j, j}^{(n)}(h) a_{k, k}^{(n)}(h)}=\rho_{j, k}(h)+o(1 / \sqrt{n})$ for $j, k=1, \ldots, q, h \in \mathbf{Z}$.

(G4) the $(p, p)$-matrix $\Phi(0) \equiv\left\{\rho_{j, k}(0): j, k=1, \ldots, q\right\}$ is regular.

Under Grenander's conditions, there exists a Hermitian matrix function $\mathbf{M}(\lambda)=$ $\left\{M_{j, k}(\lambda): j, k=1, \ldots, q\right\}$ with positive semidefinite increments such that

$$
\Phi(h)=\int_{-\pi}^{\pi} e^{i h \lambda} d \mathbf{M}(\lambda) .
$$

$\mathbf{M}(\lambda)$ is the regression spectral measure of $\{Z(t)\}$. Next we discuss the asymptotics for sample versions of $\operatorname{cov}(X, Z)$ and $\operatorname{cov}\{X X, Z\}$. For this we need the following assumption, There exists constant $b>0$ such that

$$
\operatorname{det}\left\{f_{X}(\lambda)\right\} \geq b,
$$

where $f_{X}(\lambda)$ is the spectral density matrix of $\{X(t)\}$.

Theorem 3.1. Under Grenander's conditions and the assumption

$$
\sqrt{n}\left\{\widehat{A}_{j, k}-A_{j, k}\right\} \stackrel{\oplus}{\longrightarrow} N\left(0, \Omega_{j, k}\right)
$$

where the $\left(j^{\prime}, k^{\prime}\right)$-th element of $\Omega_{j, k}$ is given by

$$
V\left(j, k: j^{\prime}, k^{\prime}\right)=2 \pi \int_{-\pi}^{\pi} f_{j j^{\prime}}(\lambda) d M_{k, k^{\prime}}(\lambda)
$$

Theorem 3.2. Under Grenander's conditions and the assumption

$$
\sqrt{n}\left\{\widehat{B}_{j, m, k}-B_{j, m, k}\right\} \stackrel{\oplus}{\rightarrow} N\left(0, \Omega_{j, m, k}\right),
$$

where $\Omega_{j, m, k}=\left\{V\left(j, m, k: j^{\prime}, m^{\prime}, k^{\prime}\right)\right\}$ with

$$
\begin{aligned}
V\left(j, m, k: j^{\prime}, m^{\prime}, k^{\prime}\right)=2 \pi \int_{-\pi}^{\pi} & {\left[\int_{-\pi}^{\pi}\left\{f_{j m^{\prime}}\left(\lambda-\lambda_{1}\right) f_{m j^{\prime}}\left(\lambda_{1}\right)+f_{j j^{\prime}}\left(\lambda-\lambda_{1}\right) f_{m m^{\prime}}\left(\lambda_{1}\right)\right\} d \lambda_{1}\right.} \\
& \left.+\iint_{-\pi}^{\pi} f_{j m j^{\prime} m^{\prime}}\left(\lambda_{1}, \lambda_{2}-\lambda, \lambda_{2}\right) d \lambda_{1} \lambda_{2}\right] d M_{k, k^{\prime}}(\lambda) .
\end{aligned}
$$

\subsection{Numerical Studies for Nonstochastic Exogenous Variables}

Letting $\{X(t)\}$ and $\{Z(t)\}$ be scalar processes, we investigate an influence of non-stochastic process $\{Z(t)\}$ on $\{X(t)\}$. The figures below show influence of harmonic trends $\{Z(t)\}$ on $V\left(j, m, k: j^{\prime}, m^{\prime}, k^{\prime}\right)$ in $\Omega_{j, m, k}$. In these cases $V\left(j, m, k: j^{\prime}, m^{\prime}, k^{\prime}\right)$ measures the amount of covariance between $X X$ and $Z$. 


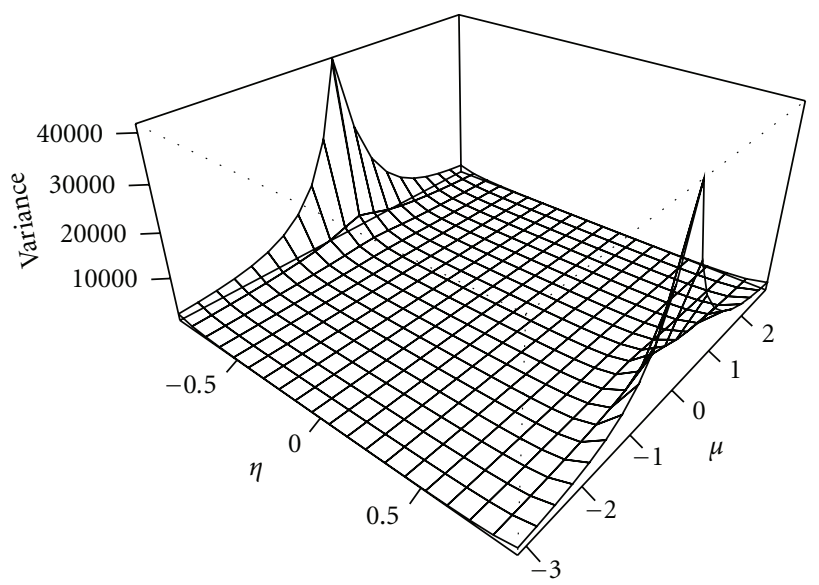

Figure 2: Values of $V\left(j, m, k: j^{\prime}, m^{\prime}, k^{\prime}\right)$ in Theorem 3.2 for $\eta=-0.9(0.1) 0.9, \mu=-3.14(0.33) 3.14$.

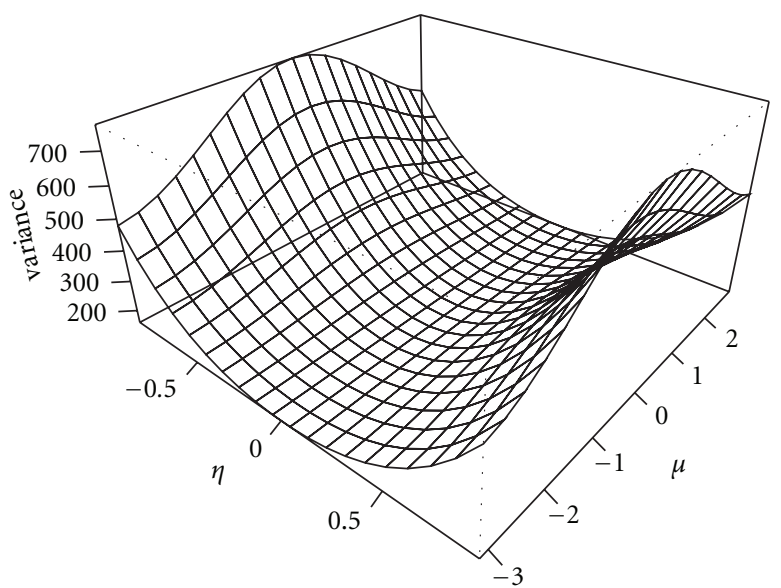

Figure 3: Values of $V\left(j, m, k: j^{\prime}, m^{\prime}, k^{\prime}\right)$ for $\eta=-0.9(0.1) 0.9, \mu=-3.14(0.33) 3.14$.

Example 3.3. Let the return process $\{X(t)\}$ and the exogenous process $\{Z(t)\}$ be generated by

$$
\begin{gathered}
X(t)=\varepsilon(t)-\eta \varepsilon(t-1), \\
Z(t)=\cos (\mu t)+\cos (0.25 \mu t),
\end{gathered}
$$

where $\varepsilon(t)^{\prime}$ s are i. i. d. $N(0,1)$ variables. Next, suppose that $\{Z(t)\}$ consists of harmonic trends with period $\mu$ and the quarter period. We plotted the graph of $V\left(j, m, k: j^{\prime}, m^{\prime}, k^{\prime}\right)$ in Figure 2.

Example 3.4. Assume that $\{X(t)\}$ and $\{Z(t)\}$ are generated by

$$
\begin{gathered}
X(t)-\eta X(t-1)=\varepsilon(t), \\
Z(t)=\cos (\mu t)+\cos (0.25 \mu t) .
\end{gathered}
$$


We observe that there exist two peaks in Figure 3. If $\mu \approx 0$ and $|\eta| \approx 1, V\left(j, m, k: j^{\prime}, m^{\prime}, k^{\prime}\right)$ increases rapidly. For further study it may be noted that Cheng et al. [4] discussed statistical estimation of generalized multiparameter likelihood models. Although these models are for independent samples, there is some possibility to apply them to our portfolio problem.

\section{Appendix}

This section provides the proofs of theorems.

Proof of Theorem 2.1. Our setting includes the exogenous variables. Although Shiraishi and Taniguchi's [1] setting does not include them, we can prove the theorem in line with Shiraishi and Taniguchi [1].

Let

$$
\begin{gathered}
\tilde{c}^{a_{2} a_{3}}=\frac{1}{n} \sum_{s=1}^{n}\left(Y_{a_{2}}-\mu_{a_{2}}\right)\left(Y_{a_{3}}-\mu_{a_{3}}\right), \\
\tilde{c}^{a_{6} a_{7} a_{8}}=\frac{1}{n} \sum_{s=1}^{n}\left(Y_{a_{6}}-\mu_{a_{6}}\right)\left(Y_{a_{7}}-\mu_{a_{7}}\right)\left(Y_{a_{8}}-\mu_{a_{8}}\right) .
\end{gathered}
$$

From Fuller [5], it is easy to see that

$$
\begin{aligned}
& \left(\widehat{c}^{a_{6}}-\mu_{a_{6}}\right)\left(\widehat{c}^{a_{7}}-\mu_{a_{7}}\right)\left(\widehat{c}^{a_{8}}-\mu_{a_{8}}\right)=o_{p}\left(\frac{1}{\sqrt{n}}\right), \\
& \widetilde{c}^{a_{6} a_{7}}\left(\widehat{c}^{a_{8}}-\mu_{a_{8}}\right)=c^{a_{6} a_{7}}\left(\widehat{c}^{a_{8}}-\mu_{a_{8}}\right)+o_{p}\left(\frac{1}{\sqrt{n}}\right),
\end{aligned}
$$

where

$$
1 \leq a_{6}, a_{7} \leq p, \quad p+1 \leq a_{8}+p+q .
$$

Then we can see that

$$
\widehat{c}^{a_{6} a_{7} a_{8}}=\widetilde{c}^{a_{6} a_{7} a_{8}}-\sum_{k=6}^{8 *} c^{a_{j_{k}} a_{i_{k}}}\left(\widehat{c}^{a_{k}}-\mu_{a_{k}}\right)+o_{p}\left(\frac{1}{\sqrt{n}}\right),
$$

where $\sum_{k=6}^{8 *}$ is the sum over $k=6,7,8$ with $i_{k}$ and $j_{k} \in\{6,7,8\}$ satisfying $i_{k}<j_{k} ; k \neq i_{k}, j_{k}$.

Hence it follows that

$$
\begin{aligned}
n \operatorname{Cov}\left(\widehat{c}^{a_{6} a_{7} a_{8}}-c^{a_{6} a_{7} a_{8}}\right)\left(\widehat{c}^{a_{6^{\prime}} a_{7^{\prime}} a_{8^{\prime}}}-c^{a_{6^{\prime}} a_{7^{\prime}} a_{8^{\prime}}}\right) \\
=n \operatorname{cum}\left\{\widehat{c}^{a_{6} a_{7} a_{8}}-\widehat{c}^{a_{6^{\prime}} a_{7^{\prime}} a_{8^{\prime}}}\right\} \\
\quad-n \sum_{k=6}^{8 *} \operatorname{cum}\left\{c^{a_{i k} a_{j_{k}}}\left(\widehat{c}^{a_{k}}-\mu_{a_{k}}\right), \widetilde{c}^{a_{6^{\prime}} a_{7^{\prime}} a_{8^{\prime}}}\right\}-n \sum_{k^{\prime}=6}^{8 *} \operatorname{cum}\left\{\widetilde{c}^{a_{6} a_{7} a_{8}}, c^{\left.a_{k^{\prime}} a_{k_{k^{\prime}}}\left(\widehat{c}^{a_{k^{\prime}}}-\mu_{a_{k^{\prime}}}\right)\right\}}\right. \\
\quad+n \sum_{k=6}^{8 *} \sum_{k^{\prime}=6}^{8 *} \operatorname{cum}\left\{c^{a_{i_{k}} a_{j_{k}}}\left(c^{a_{k}}-\mu_{a_{k}}\right) c^{a_{i_{k^{\prime}}} a_{k^{\prime}}}\left(c^{a_{k^{\prime}}}-\mu_{a_{k^{\prime}}}\right)\right\}+o(1) .
\end{aligned}
$$


In what follows we assume that $\left(a_{i_{1}}, \ldots, a_{i_{6}}\right)$ is an arbitrary permutation of $\left(a_{6}, a_{7}, a_{8}, a_{6^{\prime}}, a_{7^{\prime}}, a_{8^{\prime}}\right)$,

$$
1 \leq a_{6}, a_{7}, a_{6^{\prime}}, a_{7^{\prime}} \leq p, \quad p+1 \leq a_{8}, a_{8^{\prime}} \leq p+q .
$$

Then we get

$$
\begin{aligned}
\longrightarrow \sum_{t=-\infty}^{\infty}\{ & Q_{a_{6} a_{7} a_{8} a_{6^{\prime}} a_{7^{\prime}} a_{8^{\prime}}}^{Y}(0,0, t, t, t)+\sum_{\nu_{1}} Q_{a_{i_{1}} a_{i_{2}} a_{i_{3}} a_{i_{4}}}^{Y}(0, t, t) Q_{a_{i_{5}} a_{i_{6}}}^{Y}(t) \\
& \left.+\sum_{\nu_{2}} Q_{a_{i_{1}} a_{i_{2}} a_{i_{3}}}^{Y}(0, t) Q_{a_{i_{4}} a_{i_{5}} a_{i_{6}}}^{Y}(t, t)+\sum_{\nu_{3}} Q_{a_{i_{1}} a_{i_{2}}}^{Y}(t) Q_{a_{i_{5}} a_{i_{4}}}^{Y}(t)+Q_{a_{i_{5}} a_{i_{6}}}^{Y}(t)\right\} .
\end{aligned}
$$

By use of Fourier transform, we see that

$$
\begin{aligned}
(\mathrm{A} 1)= & 2 \pi \iiint \int_{-\pi}^{\pi} f_{a_{6} a_{7} a_{8} a_{6^{\prime}} a_{7^{\prime}} a_{8^{\prime}}}\left(\lambda_{1}, \lambda_{2}, \lambda_{3}, \lambda_{4},-\lambda_{3},-\lambda_{4}\right) d \lambda_{1} \cdots d \lambda_{4} \\
& +2 \pi \iiint_{-\pi}^{\pi} \sum_{\nu_{1}} f_{a_{i_{1}} a_{i_{2}} a_{i_{3}} a_{i_{4}}}\left(\lambda_{1}, \lambda_{2}, \lambda_{3}\right) f_{a_{i_{5}} a_{i_{6}}}\left(-\lambda_{2}-\lambda_{3}\right) d \lambda_{1} d \lambda_{2} d \lambda_{3} \\
& +2 \pi \iiint_{-\pi}^{\pi} \sum_{\nu_{2}} f_{a_{i_{1}} a_{i_{2}} a_{i_{3}}}\left(\lambda_{1}, \lambda_{2}\right) f_{a_{i_{4}} a_{i_{5}} a_{i_{6}}}\left(\lambda_{3},-\lambda_{2}-\lambda_{3}\right) d \lambda_{1} d \lambda_{2} d \lambda_{3} \\
& +2 \pi \iiint_{-\pi}^{\pi} \sum_{v_{3}} f_{a_{i_{1}} a_{i_{2}}}\left(\lambda_{1}\right) f_{a_{i_{3}} a_{i_{4}}}\left(\lambda_{2}\right) f_{a_{i_{5}} a_{i_{6}}}\left(-\lambda_{1}-\lambda_{2}\right) d \lambda_{1} d \lambda_{2} .
\end{aligned}
$$

The other asymptotic covariances are similarly evaluated. Finally, it suffices to prove the asymptotic normality of $\sqrt{n}(\widehat{\theta}-\theta)$. For this we prove

$$
\operatorname{cum}\left\{\sqrt{n}\left(\widehat{\theta}^{a_{1}}-\theta^{a_{1}}\right), \ldots, \sqrt{n}\left(\widehat{\theta}^{a_{j}}-\theta^{a_{j}}\right)\right\} \longrightarrow 0, \quad j \geq 3
$$

where $\widehat{\theta}^{a_{j}}$ and $\theta^{a_{j}}$ are the $i$ th component of $\widehat{\theta}$ and $\theta$, respectively. Let

$$
\widehat{\theta}^{a_{j}}-\theta^{a_{j}}= \begin{cases}\hat{c}^{b_{1}}-c^{b_{1}} & \text { if } i=1, \ldots, j_{1} \\ \hat{c}^{b_{2} b_{3}}-c^{b_{2} b_{3}} & \text { if } i=j_{1}+1, \ldots, j_{1}+j_{2} \\ \widehat{c}^{b_{4} b_{5}}-c^{b_{4} b_{5}} & \text { if } i=j_{1}+j_{2}+1, \ldots, j_{1}+j_{2}+j_{3} \\ \widehat{c}^{b_{6} b_{7} b_{8}}-c^{b_{6} b_{7} b_{8}} & \text { if } i=j_{1}+j_{2}+j_{3} \ldots, j_{1}+j_{2}+j_{3}+j_{4}(=j) .\end{cases}
$$


Then, similarly as in Shiraishi and Taniguchi [1] we can see that

$$
\begin{aligned}
\operatorname{cum}\left\{\sqrt{n}\left(\widehat{\theta}^{a_{1}}-\theta^{a_{1}}\right), \ldots, \sqrt{n}\left(\widehat{\theta}^{a_{j}}-\theta^{a_{j}}\right)\right\} & =n^{n / 2} \operatorname{cum}\left\{\widehat{\theta}^{a_{1}}, \ldots, \widehat{\theta}^{a_{1}+1}, \ldots, \widehat{\theta}^{a_{j_{1}+j_{2}+j_{3}+j_{4}}}\right\} \\
& =O\left(n^{1-j / 2}\right) \quad \text { for } j \geq 3,
\end{aligned}
$$

which implies the asymptotic normality of $\sqrt{n}(\widehat{\theta}-\theta)$.

Proof of Theorem 3.1. We write $\sqrt{\sum_{t=1}^{n} Z_{k}^{2}(t)}$ by $d_{t, k}(n)$. Then,

$$
\begin{aligned}
\mathbb{E}\left[\widehat{A}_{j, k}\right] & =\frac{\mu_{j} \sum_{t=1}^{n} 1 \cdot Z_{k}(t)}{\sqrt{n} d_{t, k}(n)} \quad(\text { by }(\mathrm{G} 3)) \\
& =\mu_{j} \int_{-\pi}^{\pi} d M_{0, k}(\lambda)+o\left(\frac{1}{\sqrt{n}}\right) \stackrel{\text { say }}{=} A_{j, k}
\end{aligned}
$$

which leads to

$$
\mathbb{E}\left[\widehat{A}_{j, k}\right]=A_{j, k}+o\left(\frac{1}{\sqrt{n}}\right)
$$

Next we evaluate the covariance:

$$
\begin{aligned}
\operatorname{Cov} & \left(\sqrt{n}\left\{\widehat{A}_{j, k}-A_{j, k}\right\}, \sqrt{n}\left\{\widehat{A}_{j^{\prime}, k^{\prime}}-A_{j^{\prime}, k^{\prime}}\right\}\right) \\
& =n \mathbb{E}\left[\left\{\left(\widehat{A}_{j, k}-A_{j, k}\right)-\mathbb{E}\left[\widehat{A}_{j, k}-A_{j, k}\right]\right\}\left\{\left(\widehat{A}_{j^{\prime}, k^{\prime}}-A_{j^{\prime}, k^{\prime}}\right)-\mathbb{E}\left[\widehat{A}_{j^{\prime}, k^{\prime}}-A_{j^{\prime}, k^{\prime}}\right]\right\}\right] \\
& =n \mathbb{E}\left[\left\{\left(\widehat{A}_{j, k}-A_{j, k}\right)+o\left(\frac{1}{\sqrt{n}}\right)\right\}\left\{\left(\widehat{A}_{j^{\prime}, k^{\prime}}-A_{j^{\prime}, k^{\prime}}\right)+o\left(\frac{1}{\sqrt{n}}\right)\right\}\right] \\
& =n \mathbb{E}\left[\left(\widehat{A}_{j, k}-A_{j, k}\right)\left(\widehat{A}_{j^{\prime}, k^{\prime}}-A_{j^{\prime}, k^{\prime}}\right)\right]+o(1) \\
& =\sum_{t=1}^{n} \sum_{s=1}^{n} R_{j, j^{\prime}}(s-t) \frac{Z_{k}(t) Z_{k^{\prime}}(s)}{d_{t, k}(n) d_{s \cdot k^{\prime}}(n)} \\
& =\sum_{l=-n+1}^{n-1} R_{j, j^{\prime}}(l) \sum_{s=1}^{n} \frac{Z_{k}(s-l)-l \leq n}{d_{s-l, k}(n) d_{s \cdot k^{\prime}}(n)} \\
& \longrightarrow 2 \pi \int_{-\pi}^{\pi} f_{j j^{\prime}}(\lambda) d M_{k, k^{\prime}}(\lambda)=V\left(j, k: j^{\prime}, k^{\prime}\right),
\end{aligned}
$$

where $R_{j, j^{\prime}}(s-t)$ is the covariance function of $X_{j}(t)$ and $X_{j^{\prime}}(s)$.

The asymptotic normality of $\sqrt{n}\left(\widehat{A}_{j, k}-A_{j, k}\right)$ can be shown if we prove

$$
\operatorname{cum}\left\{\sqrt{n}\left(\widehat{A}_{j_{1}, k_{1}}-A_{j_{1}, k_{1}}\right), \ldots, \sqrt{n}\left(\widehat{A}_{j_{l}, k_{l}}-A_{j_{l}, k_{l}}\right)\right\} \rightarrow 0, \quad l \geq 3
$$


Similarly as in Theorem 5.11.1 of Brillinger [6], we can see that

$$
\begin{aligned}
\operatorname{cum} & \left\{\sqrt{n}\left(\widehat{A}_{j_{1}, k_{1}}-A_{j_{1}, k_{1}}\right), \ldots, \sqrt{n}\left(\widehat{A}_{j_{l}, k_{l}}-A_{j_{l}, k_{l}}\right)\right\} \\
= & n^{l / 2} \operatorname{cum}\left\{\widehat{A}_{j_{1}, k_{1}}, \ldots, \widehat{A}_{j_{l}, k_{l}}\right\} \\
= & O\left(n^{1-l / 2}\right), \quad \text { for } l \geq 3 .
\end{aligned}
$$

Proof of Theorem 3.2. First, it is seen that

$$
\begin{aligned}
\lim _{n \rightarrow \infty} \mathbb{E}\left[\widehat{B}_{j, m, k}\right] & =\lim _{n \rightarrow \infty} R_{j, m}(0) \frac{\sum_{t=1}^{n} Z_{k}(t)}{\sqrt{n} d_{t, k}(n)} \\
& \longrightarrow R_{j, m}(0) \int_{-\pi}^{\pi} d M_{0, k}(\lambda) \stackrel{\text { say }}{=} B_{j, m, k} \quad \text { (by (G3)). }
\end{aligned}
$$

We can evaluate the covariance as follows:

$$
\begin{aligned}
& \operatorname{Cov}(\left.\sqrt{n}\left\{\widehat{B}_{j, k, m}-B_{j, k, m}\right\}, \sqrt{n}\left\{\widehat{B}_{j^{\prime}, k^{\prime}, m^{\prime}}-B_{j^{\prime}, k^{\prime}, m^{\prime}}\right\}\right) \\
&= n \mathbb{E}\left[\left(\widehat{B}_{j, k, m}-B_{j, k, m}\right)\left(\widehat{B}_{j^{\prime}, k^{\prime}, m^{\prime}}-B_{j^{\prime}, k^{\prime}, m^{\prime}}\right)\right] \\
&= n \mathbb{E}\left[\widehat{B}_{j, k, m} \widehat{B}_{j^{\prime}, k^{\prime}, m^{\prime}}-B_{j, k, m} B_{j^{\prime}, k^{\prime}, m^{\prime}}\right] \\
&= \sum_{t=1}^{n} \sum_{s=1}^{n} \operatorname{Cov}\left(X_{j}(t) X_{m}(t), X_{j^{\prime}}(s) X_{m^{\prime}}(s)\right) \int_{-\pi}^{\pi} d M_{k, k^{\prime}}(\lambda)+o(1) \\
&= \int_{-\pi}^{\pi} \sum_{l=-\infty}^{\infty}\left\{\operatorname{cum}_{j, m, j^{\prime}, m^{\prime}}(0, l, l)+R_{j, m^{\prime}}(l) R_{m, j^{\prime}}(l)+R_{j, j^{\prime}}(l) R_{m, m^{\prime}}(l)\right\} e^{-i l \lambda} d M_{k, k^{\prime}}(\lambda) \\
& \longrightarrow 2 \pi \int_{-\pi}^{\pi}\left[\int_{-\pi}^{\pi}\left\{f_{j m^{\prime}}\left(\lambda-\lambda_{1}\right) f_{m j^{\prime}}\left(\lambda_{1}\right)+f_{j j^{\prime}}\left(\lambda-\lambda_{1}\right) f_{m m^{\prime}}\left(\lambda_{1}\right)\right\} d \lambda_{1}\right. \\
&\left.\quad+2 \pi \iint_{-\pi}^{\pi} f_{j m j^{\prime} m^{\prime}}\left(\lambda_{1}, \lambda_{2}+\lambda,-\lambda_{2}\right) d \lambda_{1} d \lambda_{2}\right] d M_{k, k^{\prime}}(\lambda) \\
&=V\left(j, m, k: j^{\prime}, m^{\prime}, k^{\prime}\right) .
\end{aligned}
$$

Next we derive the asymptotic normality of $\sqrt{n}\left(\widehat{B}_{j, m, k}-B_{j, m, k}\right)$. For this we prove

$$
\operatorname{cum}\left\{\sqrt{n}\left(\widehat{B}_{j_{1}, k_{1}, m_{1}}-B_{j_{1}, k_{1}, m_{1}}\right), \ldots, \sqrt{n}\left(\widehat{B}_{j_{l}, k_{l}, m_{l}}-B_{j_{l}, k_{l}, m_{l}}\right)\right\} \rightarrow 0, \quad l \geq 3
$$


Similarly as in Theorem 5.11.1 of Brillinger [6], it is shown that

$$
\begin{aligned}
\operatorname{cum} & \left\{\sqrt{n}\left(\widehat{B}_{j_{1}, m_{1}, k_{1}}-B_{j_{1}, m_{1}, k_{1}}\right), \ldots, \sqrt{n}\left(\widehat{B}_{j_{l}, m_{l}, k_{l}}-B_{j_{l}, m_{l}, k_{l}}\right)\right\} \\
= & n^{l / 2} \operatorname{cum}\left\{\widehat{B}_{j_{1}, m_{1}, k_{1}}, \ldots, \widehat{B}_{j_{l}, m_{l}, k_{l}}\right\} \\
= & O\left(n^{1-l / 2}\right),
\end{aligned}
$$

which proves the asymptotic normality.

\section{Acknowledgments}

The authors thank Professor Cathy Chen and two referees for their kind comments.

\section{References}

[1] H. Shiraishi and M. Taniguchi, "Statistical estimation of optimal portfolios depending on higher order cumulants," Annales de l'ISUP, vol. 53, no. 1, pp. 3-18, 2009.

[2] D. M. Keenan, "Limiting behavior of functionals of higher-order sample cumulant spectra," The Annals of Statistics, vol. 15, no. 1, pp. 134-151, 1987.

[3] M. Taniguchi, "On estimation of the integrals of the fourth order cumulant spectral density," Biometrika, vol. 69, no. 1, pp. 117-122, 1982.

[4] M.-Y. Cheng, W. Zhang, and L.-H. Chen, "Statistical estimation in generalized multiparameter likelihood models," Journal of the American Statistical Association, vol. 104, no. 487, pp. 1179-1191, 2009.

[5] W. A. Fuller, Introduction to Statistical Time Series, John Wiley \& Sons, New York, NY, USA, 2nd edition, 1996.

[6] D. R. Brillinger, Time Series:Data Analysis and Theory, Holden-Day, San Francisco, Calif, USA, 2nd edition, 1981. 


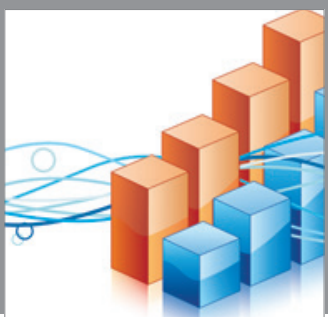

Advances in

Operations Research

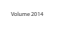

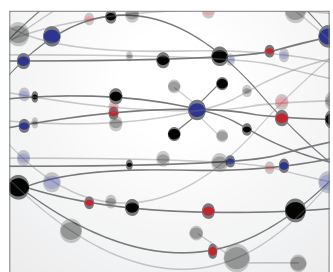

\section{The Scientific} World Journal
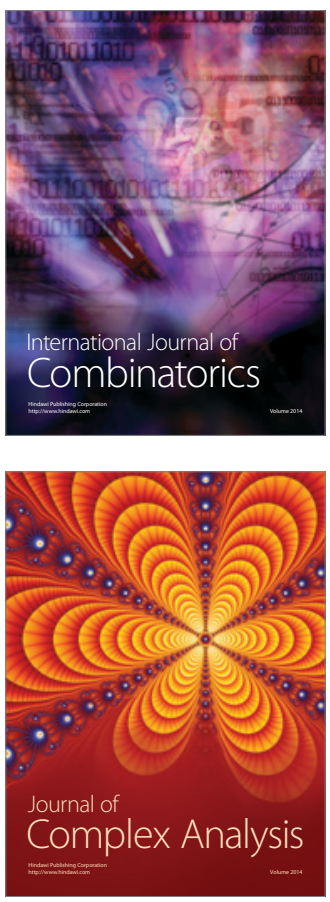

International Journal of

Mathematics and

Mathematical

Sciences
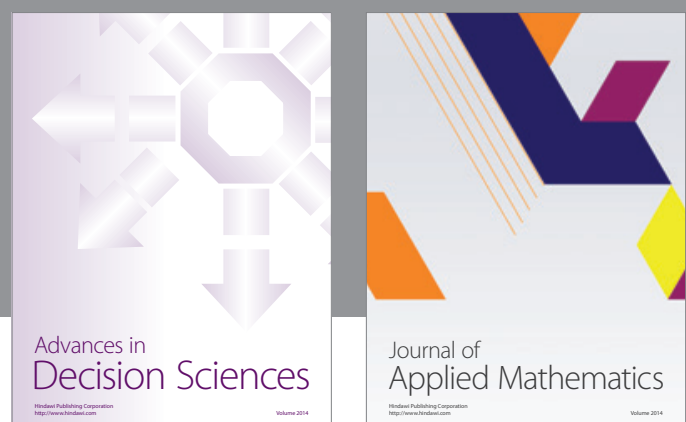

Journal of

Applied Mathematics
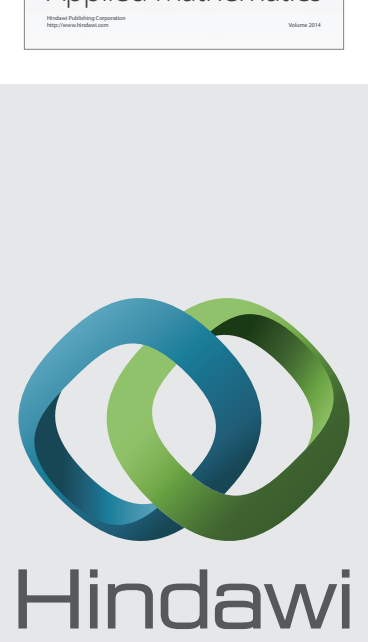

Submit your manuscripts at http://www.hindawi.com
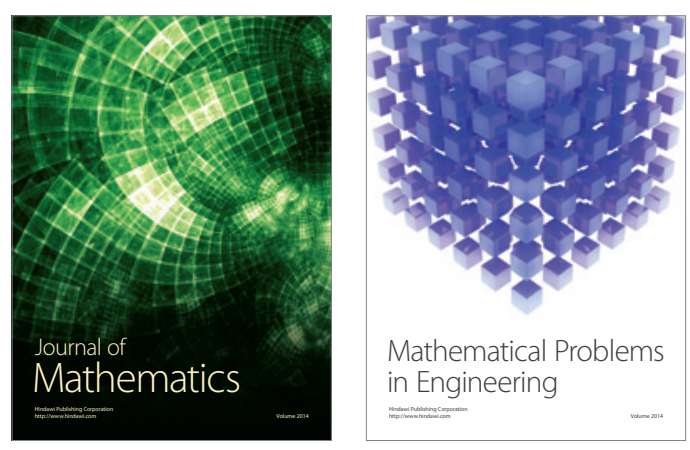

Mathematical Problems in Engineering
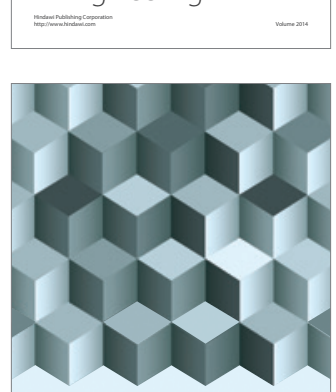

Journal of

Function Spaces
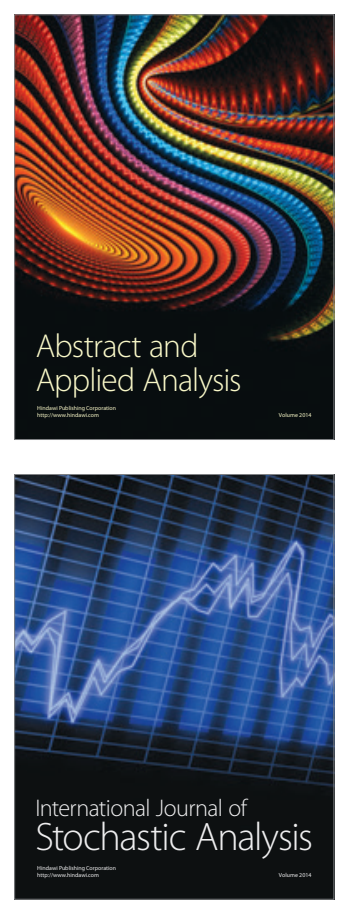

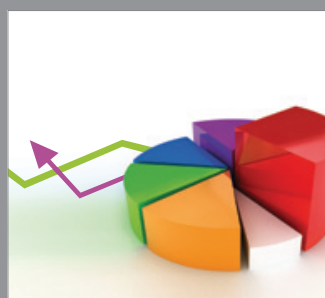

ournal of

Probability and Statistics

Promensencen
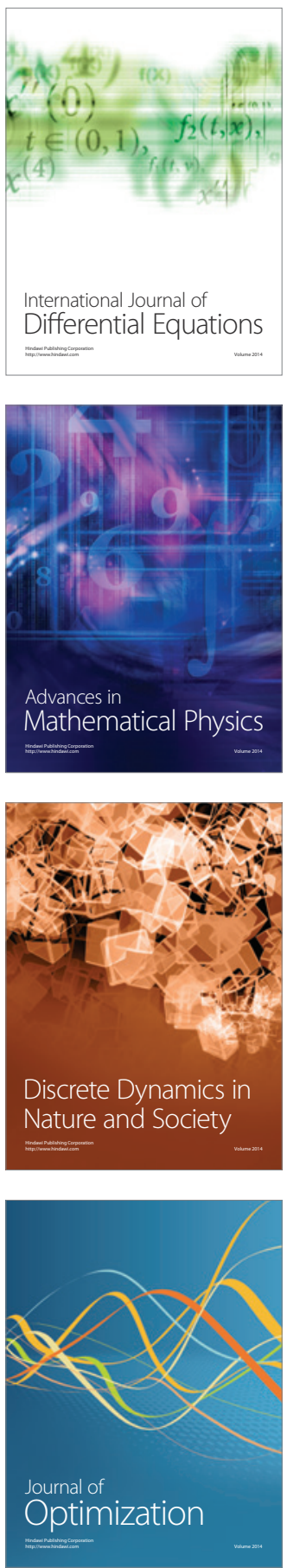